\title{
Preparation and property investigation of multi-walled carbon nanotube (MWCNT)/epoxy composite films as high-performance electric heating (resistive heating) element
}

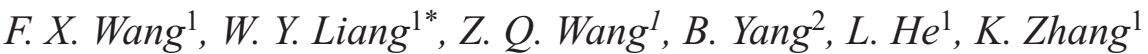 \\ ${ }^{1}$ College of Aerospace and Civil Engineering, Harbin Engineering University, 150001 Harbin, China \\ ${ }^{2}$ School of Mechanical and Power Engineering, East China University of Science and Technology, 200237 Shanghai, China
}

Received 26 September 2017; accepted in revised form 21 November 2017

\begin{abstract}
A series of multi-walled carbon nanotube (MWCNT)/epoxy composite films with a thickness of $\sim 700 \mu \mathrm{m}$ is prepared by a sequential process of premixing, post dispersing, film casting, and thermal curing. The effects of the physical shear dispersion on the properties of conductive polymer composites as the electric heating element are investigated. The scanning electron microscope (SEM) images show that highly efficient conductive networks form with shear dispersions of MWCNTs in the polymer matrix. The electrical resistivity decreases sharply from $\sim 10^{15} \Omega \cdot \mathrm{cm}$ for the neat epoxy resin to $\sim 10^{2} \Omega \cdot \mathrm{cm}$ for the composite film with $2.0 \mathrm{wt} \%$ MWCNTs in accordance with the percolation behaviour, and a low percolation threshold of $\sim 0.018 \mathrm{wt} \%$ is fitted. The electric heating behaviour of the composite film is observed at a low MWCNT content of $0.05 \mathrm{wt} \%$ due to the high electrical conductivity. For the composite film with $2.0 \mathrm{wt} \% \mathrm{MWCNTs}$, an equilibrium temperature of $115^{\circ} \mathrm{C}$ is reached at an applied voltage of $40 \mathrm{~V}$ within $30 \mathrm{~s}$. The excellent electric heating behaviour, including the rapid temperature response, electric heating efficiency, and operational stability, is primarily related to the conductive two-dimensional networks consisting of MWCNTs and the thermodynamically stable polymer matrix.
\end{abstract}

Keywords: polymer composites, shear dispersion, electrical properties, electric heating behaviour

\section{Introduction}

Carbon-based fillers, including carbon black (CB), graphene, carbon nanofibres (CNFs), and carbon nanotubes (CNTs), have been applied to improve the mechanical properties, electrical and thermal conductivities of polymer composites due to their unique nanostructures and outstanding properties [1-6]. In particular, one-dimensional CNTs with large aspect ratios and a high electrical conductivity can form continuously conductive networks with a fairly low CNT content in insulating polymer matrixes [7]. Therefore, CNTs are attractive candidates as filler materials in conductive polymer composites, and electrical energy can be converted into heat energy through electric Joule heating (resistive heating) in conductive networks consisting of CNTs. For the conductive polymer composite as the electric heating element, a polymer matrix with excellent thermodynamic stability is a prerequisite. Through the cross-linking reaction, the epoxy resin can form a thermosetting polymer with excellent mechanical properties, temperature and chemical resistance, in addition to the advantages of its easy processability and economical price. An and Jeong [8] reported graphene/epoxy composite films as electric heating elements, in which conductive networks were formed at relatively high graphene content between 1.0 and $2.0 \mathrm{wt} \%$ that were ascribed to the sheet structure of graphene. Jeong and An [9] investigated MWCNT/graphene/epoxy composite films with $5.0 \mathrm{wt} \%$ mixed carbon fillers with 
different graphene/ MWCNT compositions and found that a higher equilibrium temperature was reached at a given voltage with a higher MWCNT content in $5.0 \mathrm{wt} \%$ mixed carbon fillers due to the bridging effect of the MWCNTs.

To date, electric heating elements have been primarily made from metallic materials such as $\mathrm{Ni}-\mathrm{Cr}$ (nichrome) or Fe-Cr-Al (Kanthal); however, the main difficulties in the use of nichrome or Kanthal lie in the heavy weight, electromagnetic wave emission, difficult processability, and susceptibility to corrosion. In contrast, conductive polymer composites as the electric heating element offer a light weight, faster and more efficient heating, and corrosion resistance. Because of the range of advantages over metallic materials, the conductive polymer composites have the potential to replace them in some applications, such as in water heater, defogger, functional textile, and floor heater. Accordingly, the conductive polymer composite as the electric heating element or device has generated enormous scientific interest [10-14]. The potential of CNTs as filler has been severely limited because of their dispersion problem in the polymer matrix. The CNTs became highly aggregated due to the Van der Waals interactions among the smalldiameter nanotubes. In the laboratory, ultrasonication is the most frequently used method for CNT dispersion in low-viscosity liquids, such as acetone, ethanol, and deionized water. However, some polymers are solids, and some are high-viscosity liquids, which require the polymers to be dissolved or diluted using volatile solvents to reduce the viscosity before dispersion. Calenders, also known as 3-roll mills, utilize adjacent rollers rotating at different angular velocities and the micron-scale gap between the rollers to generate enormous shear forces. The intense shear force is exerted on the entire volume of the mixture to uniformly disperse CNTs into a polymer matrix when the mixture passes through the narrow gap with a short residence time. In the past few years, this shear dispersion has been used to disperse nanoparticles into the polymer to improve its electrical and thermal properties, as well as its mechanical strength [15-19]. Better yet, this non-solvent shear dispersion method is suitable for high-viscosity polymers.

In this paper, MWCNTs were dispersed in an epoxy matrix by using a laboratory-scale 3-roll mill (TYEE, TRS-65, China), and a series of thermally stable composite films with different MWCNT contents was fabricated. The microstructural features and electrical and thermal conductivities of the composite films were investigated as a function of the MWCNT content. In addition, the electric heating behaviour of the composite films was also evaluated as a function of the MWCNT content and applied voltage by considering the temperature response rapidity, electric heating efficiency, natural cooling properties, and operational stability.

\section{Experimental section}

\subsection{Materials}

Diglycidyl ether of bisphenol-A (DGEBA, YD-128, Kukdo Chemical (Kunshan) Co., Ltd., China), 2,3,6tetrahydro-3-methylphthalic anhydride (H-316, Chuzhou Hui-Sheng Electronic Materials Co., Ltd., China), and liquid-type imidazole (2101CN, Chuzhou Hui-Sheng Electronic Materials Co., Ltd., China) were used as the epoxy compound, curing agent and accelerator for the thermosetting epoxy matrix, respectively. Pristine MWCNTs (791431-25G, SigmaAldrich Inc., USA) with an average filament diameter of $\sim 10-15 \mathrm{~nm}$ and length of $\sim 3 \mu \mathrm{m}$, which were prepared using the catalytic chemical vapor deposition (CVD) method, were dispersed in the epoxy matrix as the conductive reinforcing nanofiller.

\subsection{Preparation of the conductive polymer composites}

Epoxy-based composite films containing different MWCNT contents $(0.01-2.0 \mathrm{wt} \%$ of the epoxy matrix) were fabricated by a sequential process of premixing, post dispersing, film casting, and thermal curing, as schematically illustrated in Figure 1a. First, the epoxy resin was heated at $80^{\circ} \mathrm{C}$ to remove the entrapped air, and then, a predetermined amount of MWCNTs was added into the resin solution and stirred at $500 \mathrm{rpm}$ for $5 \mathrm{~min}$. Second, the MWCNT/ epoxy mixtures were post dispersed using a 3-roll mill, the general configuration and dispersion principle of which are illustrated in Figure 1b. The MWCNT/epoxy mixtures were successively passed through gap settings of $\delta_{\mathrm{g}}=30,20$, and $10 \mu \mathrm{m}$, at least 10 times and, finally, $5 \mu \mathrm{m}$ at least 15 times. After processing with a 3-roll mill, the mixtures were degassed under vacuum conditions at $80^{\circ} \mathrm{C}$ for $30 \mathrm{~min}$. The curing agent and accelerator were then added into the mixtures and stirred at $50 \mathrm{rpm}$ for $5 \mathrm{~min}$. The proportions of the accelerator and curing agent to the epoxy were controlled to be $1: 80: 100$ by wt $\%$, respectively. Subsequently, the mixtures were cast on 

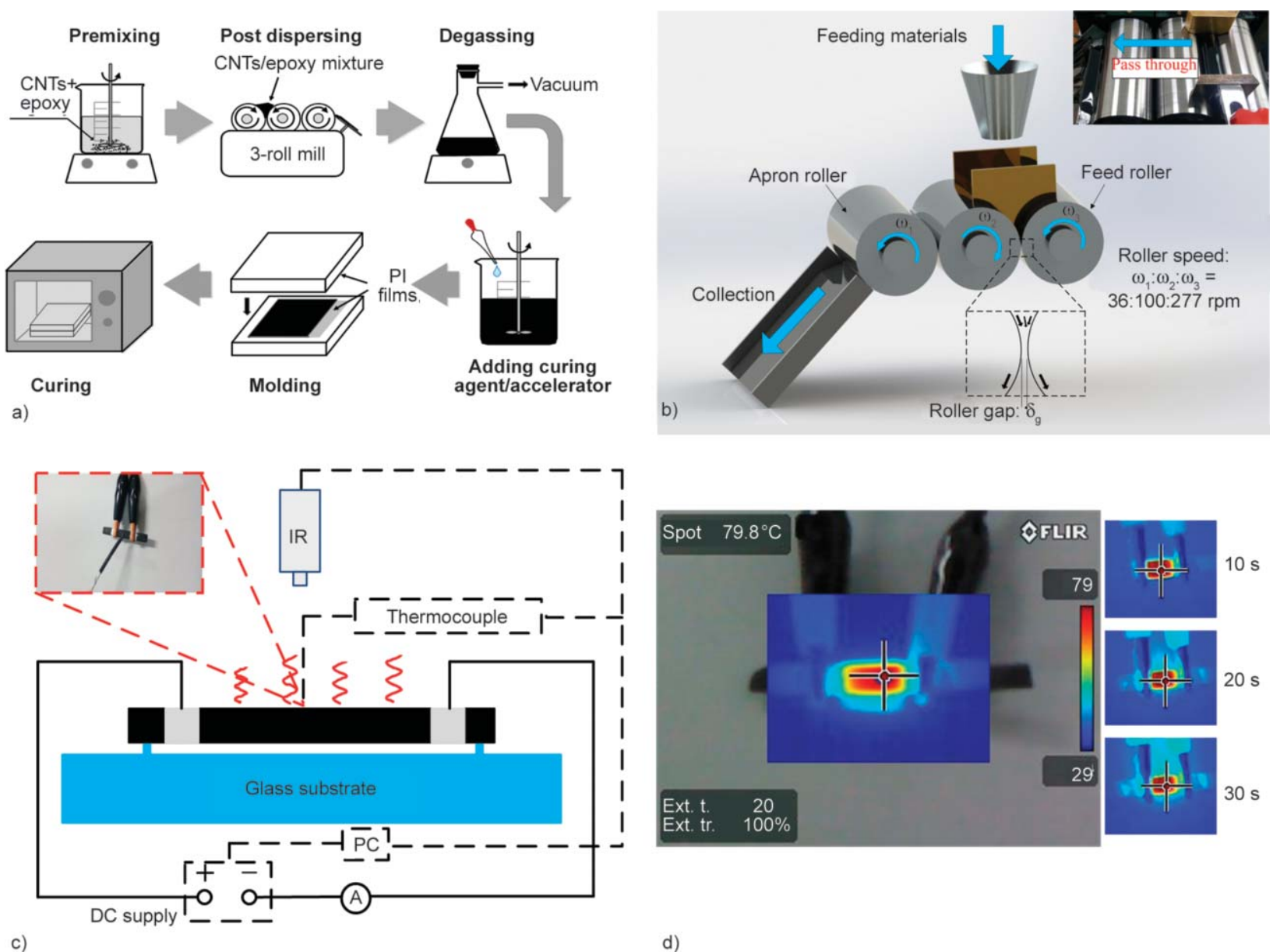

c)

d)

Figure 1. Schematic diagram illustrating (a) the preparation of the MWCNT/epoxy composite films, (b) the general configuration of a 3-roll mill and the region of high shear dispersion between the rollers, (c) electric heating behaviour test schematic diagram and (d) infrared image of the composite films

polyimide films that were pressed by an aluminium plate to control the film thickness. Finally, the mixtures were thermally cured at $100^{\circ} \mathrm{C}$ for $2 \mathrm{~h}, 120^{\circ} \mathrm{C}$ for $2 \mathrm{~h}$, and $140^{\circ} \mathrm{C}$ for $4 \mathrm{~h}$ to obtain the prepared composite films. The thickness of the final composite films was controlled to be $\sim 700 \mu \mathrm{m}$. The prepared MWCNT/epoxy composite films were referred to as CNT/EP_X, where X denotes the MWCNT weight fraction.

\subsection{Characterization}

To identify the dispersion state of the MWCNTs as well as the formation of the conductive pathways consisting of the inter-MWCNT networks within the resin matrix, the film specimens were cryogenically cracked with liquid nitrogen, and their cross sections were characterized with a scanning electron microscope (SEM, Zeiss MA15, Germany) and a fieldemission scanning electron microscope (FE-SEM, Zeiss Supra55, Germany), respectively. The electric current $(I)$ through the composite films was recorded as a function of the applied voltage $(V)$ by employing a sourcemeter (Keithley, 2700, USA). The thermal conductivity was measured by a thermal conductivity meter (XIATECH, TC 3000E, China) with the transient hot-wire method at room temperature. The electric heating behaviour of the composite films under varying applied voltages was characterized with a digital thermometer (MASTECH, MS651, China) and an infrared camera (FLIR, T420, USA). For the electrical and electric heating experiments, composite films were cut into $5.0 \mathrm{~mm}$ wide strips, and the distance between the two crocodile clips was maintained at $10.0 \mathrm{~mm}$. Highly conductive silver paint was coated on the terminals of the specimen to significantly decrease the contact resistance between the specimen and the crocodile clip. Figure 1c and 1d displays the electric heating behaviour test schematic diagram and infrared image for the characterization of the electric heating behaviour, respectively. The dynamic mechanical thermal properties of the composite specimens were examined with the aid of a 
dynamic mechanical analyser (DMA, TA Q800, USA) under tensile mode from 50 to $350^{\circ} \mathrm{C}$ at a heating rate of $5^{\circ} \mathrm{C} / \mathrm{min}$ and a frequency of $1 \mathrm{~Hz}$. The thermal stability was also examined by a thermogravimetric analyser (TGA, TA Q50, USA) under air conditions from room temperature to $600^{\circ} \mathrm{C}$ at a heating rate of $10^{\circ} \mathrm{C} / \mathrm{min}$.

\section{Results and discussion}

\subsection{Microstructural feature}

Figure 2 shows the SEM images of the composite films with different MWCNT contents. The agglomerated CNTs were observed to be separated and embedded within the epoxy matrix. On the fractured surface, some CNTs were pulled out of the matrix (inset of the Figure 2a), indicating that there were weak interfacial interactions between the CNTs and epoxy matrix. In contrast, some micron- and submicron-scale agglomeration remained, which was the result of a mismatch between the micron-scale gap setting $\left(\delta_{\mathrm{g}}\right)$ of the 3-roll mill and the nano-scale diameter of the MWCNTs. In particular, it was clearly

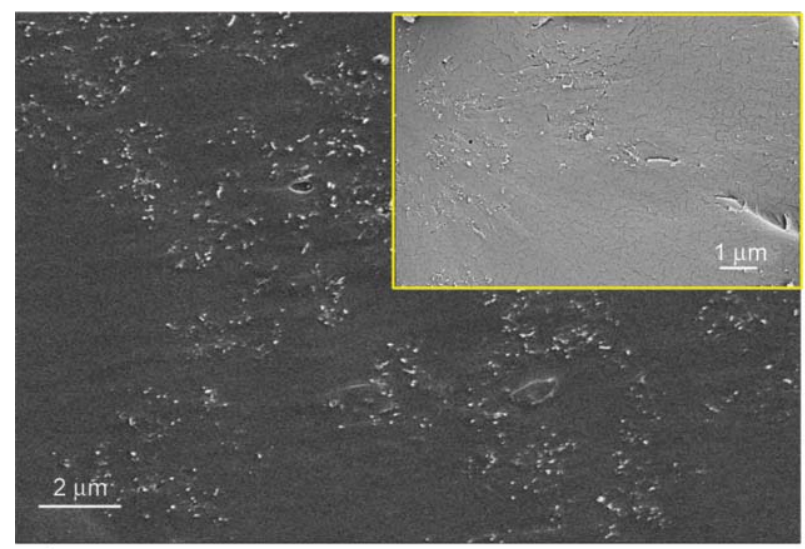

a)

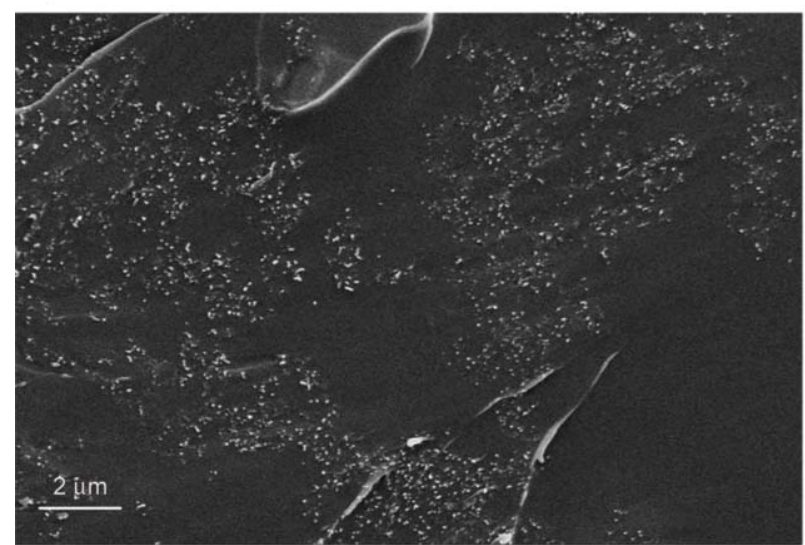

c) found that a more efficient conductive pathway was forming with the increment in the MWCNT content.

\subsection{Electrical and thermal properties}

The electrical properties of the MWCNT/epoxy composite films were investigated as functions of the filler content and applied voltage. Figure 3 shows the current-voltage $(I-V)$ curves of the neat epoxy and its composite films. For the electrically insulating epoxy film, no electric current was detected over the applied voltage range of 10-90 V. In contrast, there was a weak electric current throughout the composite film with a low MWCNT content of $0.01 \mathrm{wt} \%$, which indicates that a conductive pathway among the inter-MWCNT networks formed and that the electric charges could flow throughout the composite film. For composite films with an MWCNT content of $0.01-2.0 \mathrm{wt} \%$, the changes in the electric current with the applied voltage showed linear characteristics, and the slopes of the $I-V$ curves were steeper with higher MWCNT contents; thus, the flow properties of the electric charges with varying applied

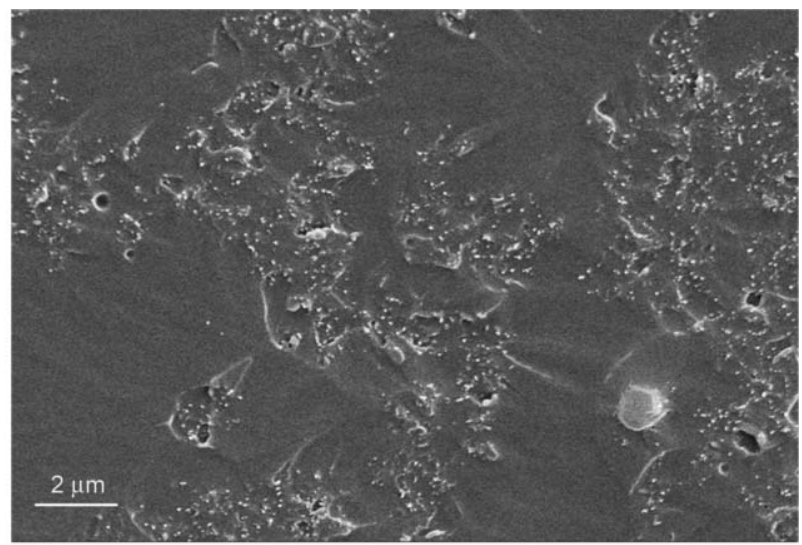

b)

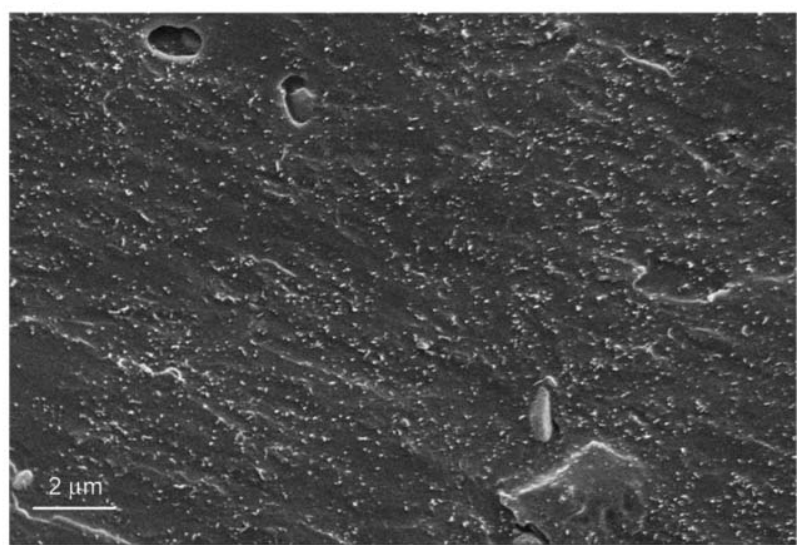

d)

Figure 2. SEM images of the composite films with different MWCNT contents: (a) CNT/EP_0.05; (d) CNT/EP_0.3; (c) CNT/EP_0.7; and (d) CNT/EP_2.0. Inset is the FE-SEM image of CNT/EP_0.05 


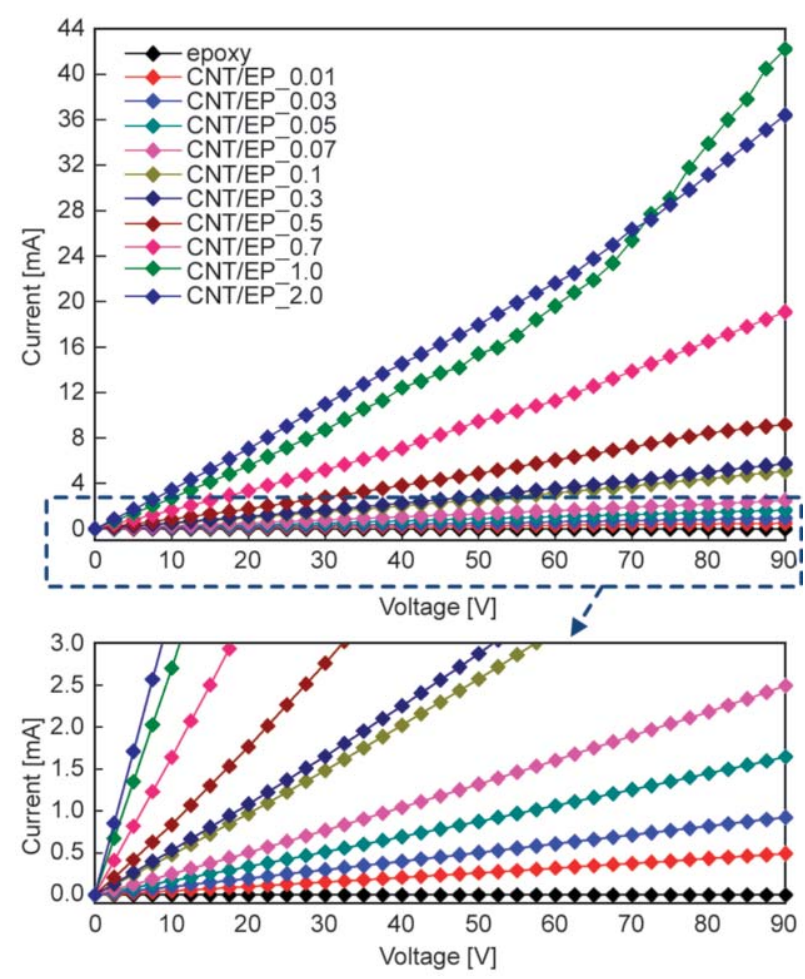

Figure 3. Current-voltage (I-V) curves for the neat epoxy and its composite films with different MWCNT contents

voltages exhibited a linear behaviour in relation to Ohm's law (Equation (1)) [11]:

$$
V=I R
$$

where $R$ is the electrical resistance of the composite film. It should be noted that, the $I-V$ curves showed non-linear behaviour in the high applied voltage range with regard to the CNT/EP 1.0 and 2.0. This non-linear behaviour can be explained by the electron transition activated by the Joule heating effect $[9,11]$. The quantum tunneling mechanism reveals that electrons can hop from a CNT to another disconnected CNT to realize electric conduction when they are close enough. The electric current flows through the direct physical contact among the CNTs in the low applied voltage range. With the higher voltage applied, the heat generated by the Joule heating increases significantly, enabling the electrons to easily hop from a CNT to an adjacent one, resulting in an increased electric current in the conductive path; thus, the $I-V$ curve deviates slightly from the linear behaviour. According to the Equation (1), the electrical resistance $(R)$ of the composite films was calculated from the inverse of the slope of $I-V$ curve in Figure 3, and the result was plotted as a function of MWCNT content in Figure 4. Furthermore, by utilizing the Equation (2) [11]:

$\rho=\frac{R A}{L}$

where $L$ is the distance between the crocodile clips and $A$ is the cross-sectional area of the composite film, the electrical resistivity $(\rho)$ was calculated and is summarized in Figure 4. The electrical resistivity decreased sharply from $\sim 10^{15} \Omega \cdot \mathrm{cm}$ for the neat epoxy resin to $\sim 10^{4} \Omega \cdot \mathrm{cm}$ for CNT/EP_0.01, and then, it decreased slightly for MWCNT contents in excess of $1.0 \mathrm{wt} \%$. For CNT/EP_2.0, the electrical resistivity was $\sim 10^{2} \Omega \cdot \mathrm{cm}, 13$ orders of magnitude lower than the value of the neat epoxy resin. The electrical resistivity was dependent on the MWCNT content, which is in reasonable agreement with the percolation theory [20]. The percolation theory predicts that there is an electrical percolation threshold at which a thorough conductive pathway forms throughout a polymer matrix, resulting in the transformation of the composite from an insulator into a semi-conductor or conductor. The electrical percolation threshold can be determined by fitting the following power law relation (Equation (3)) [20, 21]:

$\sigma \propto\left(p-p_{\mathrm{c}}\right)^{\mathrm{t}}$

where $\sigma$ is the electrical conductivity, which can be calculated from $\sigma=1 / \rho, p$ is the volume fraction of the MWCNT, $p_{\mathrm{c}}$ is the critical volume fraction or electrical percolation threshold, and $t$ is the critical exponent. The linear fitting line with $p_{\mathrm{c}}=0.014 \mathrm{vol} \%$ $(\sim 0.018 \mathrm{wt} \%)$ and $t=1.34$ correlates well with the

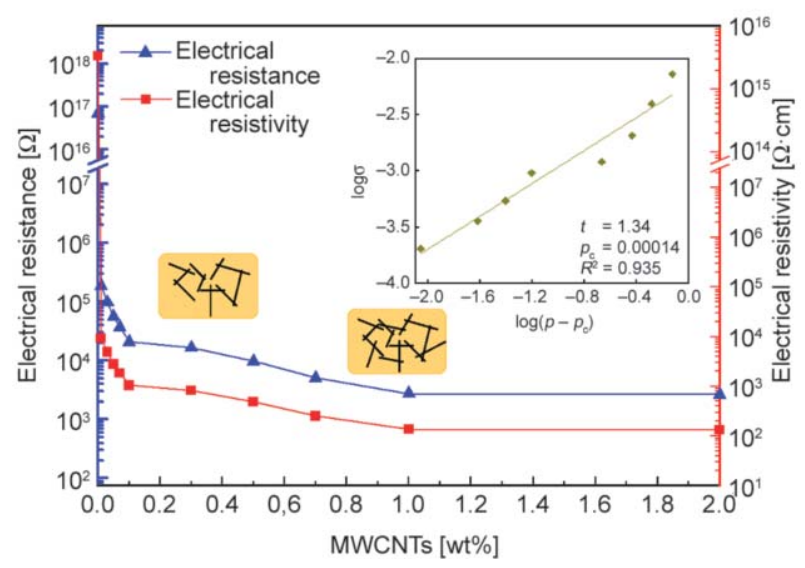

Figure 4. Electrical resistance and resistivity of the composite films as a function of the MWCNT content. Inset is a log-log plot of electrical conductivity versus MWCNT content by vol $\%$ 
experimentally measured data of $\log \sigma$ versus $\log \left(p-p_{\mathrm{c}}\right)$ (inset of Figure 4). The critical exponent $t$ generally represents the dimensionality of the system, and theoretical values of 1.3 and 1.94 have been predicted for typical 2-D and 3-D systems, respectively [20]. Hence, the $t$ value of 1.34 indicates that the MWCNTs formed a 2-D conductive network at the percolation threshold of $\sim 0.018 \mathrm{wt} \%$ MWCNTs. The low $p_{\mathrm{c}}$ value of $\sim 0.018 \mathrm{wt} \%$ stems from the uniform and random dispersion of the MWCNTs within the polymer matrix as well as the high electrical conductivity and large aspect ratio of the MWCNTs. In addition, MWCNTs dispersed in a polymer matrix can enhance not only the electrical conductivity but also the thermal conductivity of the conductive polymer composite. Figure 5 shows the influences of the MWCNT content on the thermal conductivity. In contrast to the improved electrical conductivity with the MWCNTs, in which typical percolation behaviour was observed, the thermal conductivity versus the MWCNT content curve was nearly linear, i.e. such as reported elsewhere [22]. The effects of the MWCNTs on the thermal conduction properties of the composite films were characterized by the thermal conductivity enhancement factor $\eta$ expressed as Equation (4):

$\eta=\frac{k_{\mathrm{c}}-k_{\mathrm{m}}}{k_{\mathrm{m}}} \cdot 100 \%$

where $k_{\mathrm{c}}$ and $k_{\mathrm{m}}$ represent the thermal conductivities of the composite specimens and polymer matrix, respectively. For CNT/EP_2.0, a $\eta$ value of only $~ 22 \%$ over the neat epoxy resin was observed. This limited increase was associated with the transport of thermal energy from the inter-MWCNT networks to the polymer matrix. The transport of thermal energy in

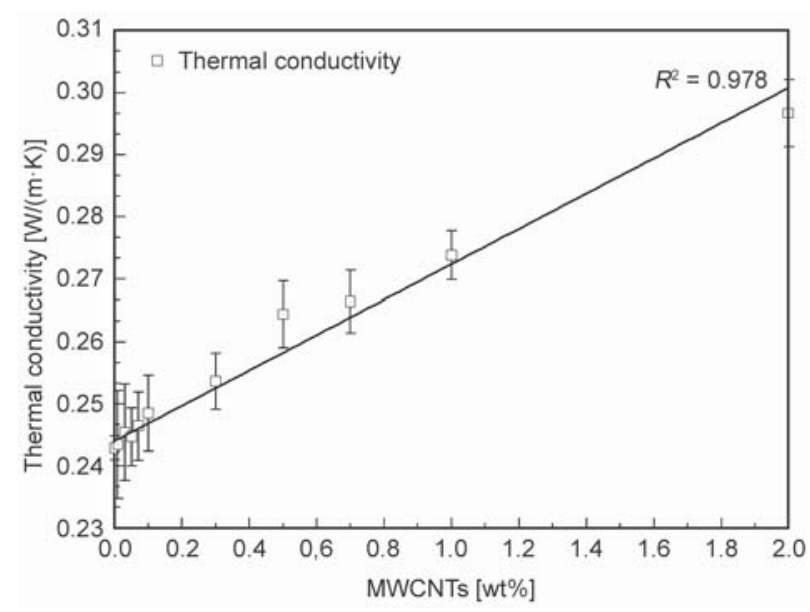

Figure 5. Thermal conductivity of the composite specimens as a function of MWCNT content
MWCNTs is related to the phonon conduction mechanism, and meanwhile, the surface scattering effect caused by the defects of CNT walls and the interfacial adhesion between CNTs and their surrounding matrix is known to significantly affect the phonon conduction. Furthermore, the physical shear force of a 3-roll mill exerted on mixtures led to the disordered, random dispersion for individual MWCNTs in the polymer matrix. In addition, there were weak interfacial interactions between the CNTs and epoxy matrix, greatly increasing the thermal contact resistance among the nanotube-nanotube and nanotube-matrix interfaces [23].

\subsection{Electric heating behaviour}

For the composite films with MWCNT contents of $0.05-2.0 \mathrm{wt} \%$, the electric heating behaviour was investigated under the applied voltages of $10-90 \mathrm{~V}$. Figure 6 displays that the surface temperature $(T)$ changes as a function of the time $(t)$ under varying applied voltages. Meanwhile, the heating and cooling rates (inset of Figure 6) under the maximum applied voltage were plotted from the derivatives of the $T-t$ curves of the composite films. It was clearly found that the electric heating behaviour of the composite films was strongly dependent on the MWCNT contents and applied voltages. In the case of CNT/ EP_0.05, the surface temperature remained at ambient temperature without any obvious change under a relatively low voltage of $10 \mathrm{~V}$, which indicates that the electric current throughout the composite film was not enough to cause a surface temperature change. However, when applied voltages above $20 \mathrm{~V}$ were applied at $5 \mathrm{~s}$, the surface temperature increased rapid$1 \mathrm{y}$, reached an equilibrium temperature within $\sim 30 \mathrm{~s}$, remained at the equilibrium temperature up to $120 \mathrm{~s}$, and then cooled down to ambient temperature within a brief period as the applied voltage was turned off at $120 \mathrm{~s}$. Compared with the graphene/epoxy [8] and graphene/MWCNT/epoxy [9] composite films, the electric heating behaviour of the MWCNT/epoxy composite films was observed at a lower filler content, and this observation was associated with the nanostructure and dispersion state of the CNTs. For the other composite films with higher MWCNT contents of $0.07-2.0 \mathrm{wt} \%$, similar electric heating behaviour was observed, and the noticeable difference was that the equilibrium temperature at an identical applied voltage was even higher with the increasing MWCNT content. For the CNT/EP_0.5 under the applied 

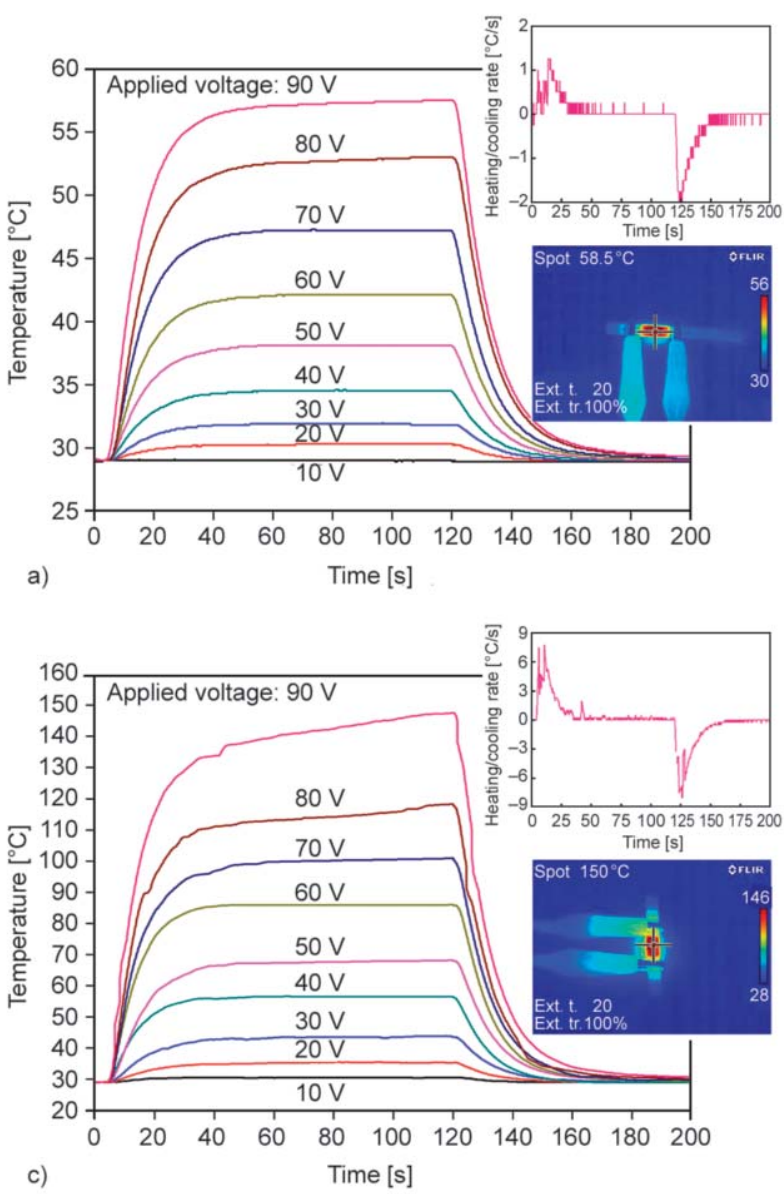
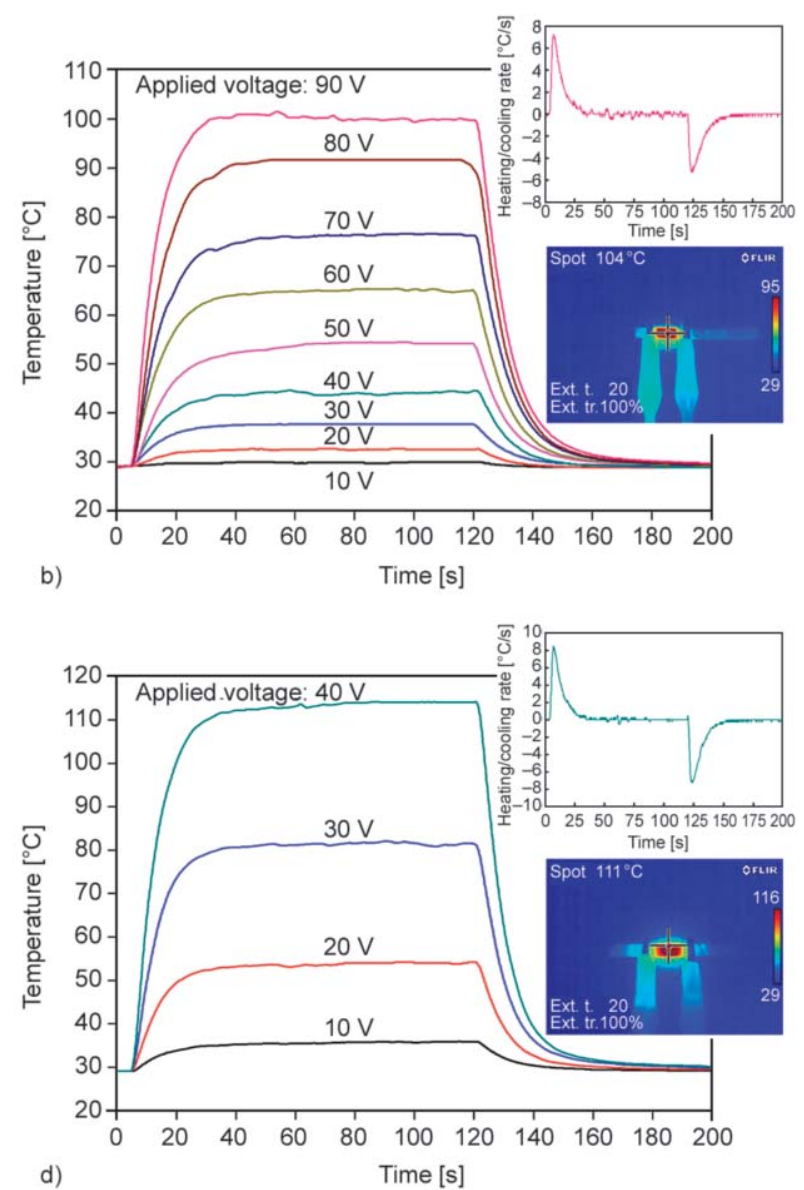

Figure 6. Surface temperature-time curves ( $T$-t curves) of the composite films at varying applied voltages from 10-90 V: (a) CNT/EP_0.05; (b) CNT/EP_0.1; (c) CNT/EP_0.5; and (d) CNT/EP_2.0. Inset is the plot of the derivative of the $T-t$ curves and the infrared image at the maximum applied voltage

voltage of $90 \mathrm{~V}$, the equilibrium temperature was not reached within $120 \mathrm{~s}$. This delay was associated with the electron transition activated by the Joule heating effect, as described above. The maximum temperature of $\sim 145^{\circ} \mathrm{C}$ easily activated electron hopping, increasing the electric current through the conductive pathway. Thus, a longer time was needed to attain the equilibrium temperature.

According to the derivative data of the $T-t$ curves, the $T-t$ curves could be defined as having three regions: the temperature growth region (5-30 s), the equilibrium temperature region (30-120 s), and the natural cooling region (120-200 s). In the temperature growth region, the surface temperature growth with time can be expressed as Equation (5) [8]:

$\frac{T_{\mathrm{t}}-T_{0}}{T_{\mathrm{e}}-T_{0}}=1-\exp \left(-\frac{t}{\tau_{\mathrm{g}}}\right)$

where $T_{0}, T_{\mathrm{e}}$, and $T_{\mathrm{t}}$ are the initial temperature, the equilibrium temperature, and an arbitrary temperature at time $t$, respectively, and $\tau_{\mathrm{g}}$ is the fitted characteristic parameter, the characteristic growth time constant.
For all the composite films showing electric heating behaviour, the $\tau_{\mathrm{g}}$ values could be calculated by fitting the data in the first region of the $T-t$ curves, and the results are summarized in Table 1. Unlike the graphene/epoxy composite films [8], the $\tau_{\mathrm{g}}$ values of the MWCNT/epoxy composite films were not strongly dependent on the MWCNT content within the experimental error. The average $\tau_{\mathrm{g}}$ value of $8.05 \pm 0.65 \mathrm{~s}$ for all the composite films was slightly higher than that of the graphene/epoxy [8] and graphene/ MWCNT/epoxy [9] composite films, while it was found to be lower than that of the MWCNT/ polyethylene composite reported in the literature [14]. This result demonstrates that the MWCNT/epoxy composite films in this paper exhibited a significantly rapid temperature response to the applied voltage.

In the equilibrium temperature region, the heat generated by the electric power equilibrates with the heat dissipated by the thermal radiation and convection in accordance with the energy conversation law. The heat dissipation by thermal radiation and convection, $h_{\mathrm{r}+\mathrm{c}}$, can be expressed as Equation (6) [8]: 
Table 1. Characteristic parameters $\left(\tau_{\mathrm{g}}, h_{\mathrm{r}+\mathrm{c}}\right.$ and $\left.\tau_{\mathrm{d}}\right)$ of the electric heating behaviour of the composite films in this article and other composite specimens reported in the literature

\begin{tabular}{|l|c|c|c|c|c|}
\hline \multicolumn{1}{|c|}{ Composite specimens } & $\begin{array}{c}\text { Filler contents } \\
{[\mathbf{w t} \% \mathbf{0}}\end{array}$ & $\begin{array}{c}\text { Voltage } \\
{[\mathbf{V}]}\end{array}$ & $\begin{array}{c}\boldsymbol{\tau}_{\mathbf{g}} \\
{[\mathbf{s}]}\end{array}$ & $\begin{array}{c}\boldsymbol{h}_{\mathbf{r}+\mathbf{c}} \\
{\left[\mathbf{m W} /{ }^{\circ} \mathbf{C}\right]}\end{array}$ & $\begin{array}{c}\boldsymbol{\tau}_{\mathbf{d}} \\
{[\mathbf{s}]}\end{array}$ \\
\hline \multirow{5}{*}{ MWCNT/epoxy } & 0.05 & $10-90$ & $9.31 \pm 0.53$ & $3.97 \pm 0.17$ & $12.61 \pm 0.73$ \\
\cline { 2 - 6 } & 0.07 & $10-90$ & $7.99 \pm 0.41$ & $4.71 \pm 1.06$ & $9.23 \pm 2.03$ \\
\cline { 2 - 6 } & 0.10 & $10-90$ & $8.06 \pm 0.56$ & $4.42 \pm 0.39$ & $10.49 \pm 0.63$ \\
\cline { 2 - 6 } & 0.30 & $10-90$ & $7.46 \pm 0.53$ & $4.66 \pm 0.46$ & $10.24 \pm 0.24$ \\
\cline { 2 - 6 } & 0.50 & $10-90$ & $8.43 \pm 0.98$ & $5.25 \pm 0.78$ & $11.68 \pm 0.73$ \\
\cline { 2 - 6 } & 0.70 & $10-60$ & $7.42 \pm 0.54$ & $4.37 \pm 0.88$ & $10.18 \pm 1.09$ \\
\cline { 2 - 6 } & 1.00 & $10-50$ & $8.29 \pm 0.66$ & $5.73 \pm 1.63$ & $11.7 \pm 0.60$ \\
\hline Graphene/epoxy [8] & 2.00 & $10-40$ & $7.42 \pm 0.74$ & $5.90 \pm 0.80$ & $10.22 \pm 1.16$ \\
\hline Graphene/MWCNT/epoxy [9] & 2.00 & $20-100$ & $5.67 \pm 1.06$ & $1.70 \pm 0.50$ & $6.67 \pm 2.22$ \\
\hline MWCNT/polyethylene [14] & 5.00 & $10-25$ & $5.20 \pm 0.70$ & $9.10 \pm 1.00$ & $2.30 \pm 0.20$ \\
\hline
\end{tabular}

$h_{\mathrm{r}+\mathrm{c}}=\frac{I_{\mathrm{s}} V_{0}}{T_{\mathrm{e}}-T_{0}}$

where $I_{\mathrm{S}}$ is the steady-state electric current and $V_{0}$ is the corresponding applied voltage. The $h_{\mathrm{r}+\mathrm{c}}$ values could be computed from the data in the equilibrium temperature region of the $T-t$ curves, as summarized in Table 1 . The $h_{\mathrm{r}+\mathrm{c}}$ value was found to increase slightly with the MWCNT content because of the higher equilibrium temperatures at an identical voltage, which intensifies the heat exchange with the composite films and its surroundings. In addition, compared with the $h_{\mathrm{r}+\mathrm{c}}$ values for other carbon nanofiller/epoxy $[8,9]$ and MWCNT/polyethylene [14] composites, the average $h_{\mathrm{r}+\mathrm{c}}$ value of $4.88 \pm 0.68 \mathrm{~mW} /{ }^{\circ} \mathrm{C}$ for the composite films in this paper is excellent, which indicates that MWCNT/epoxy composite films exhibited excellent electric heating efficiency by applying a comparatively low electric power to reach a high equilibrium temperature.

When the applied voltage was turned off at $120 \mathrm{~s}$, the electric heating behaviour disappeared, and the

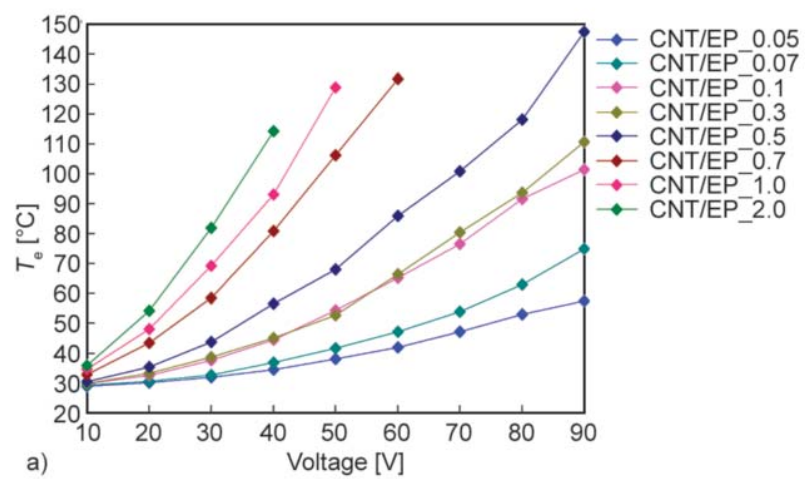

surface temperature gradually cooled down to the ambient temperature in accordance with Newton's law of cooling. In the third region, the temperature decrement with time can be expressed by the Equation (7) [8]:

$\frac{T_{\mathrm{t}}-T_{0}}{T_{\mathrm{e}}-T_{0}}=\exp \left(-\frac{t}{\tau_{\mathrm{d}}}\right)$

where $\tau_{\mathrm{d}}$ is the decay time constant. The $\tau_{\mathrm{d}}$ values were calculated by fitting the data in the third region, and they are listed in Table 1. For all the composite films, the average value of $\tau_{\mathrm{d}}$ was calculated to be $10.79 \pm 1.09 \mathrm{~s}$, which also demonstrates that the MWCNT/epoxy composite films possessed highly efficient cooling properties by cooling down to ambient temperature within a brief period.

The variations in the equilibrium temperatures $\left(T_{\mathrm{e}}\right)$ of the composite films as a function of the applied voltage $(V)$ were nonlinear, as seen in Figure $7 \mathrm{a}$. The electric power $(P)$ computational formula can be express by Equation (8) [9]:

$P=V I$

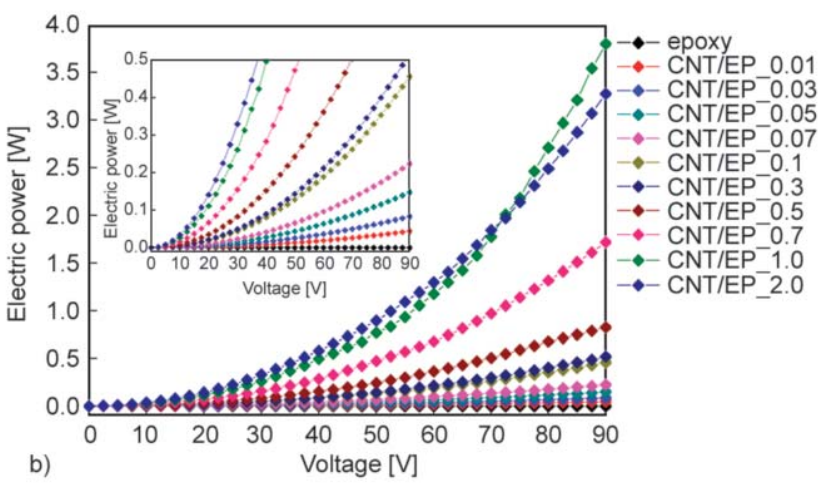

Figure 7. Changes of the (a) equilibrium temperature and (b) electric power of the composite films with different MWCNT contents as a function of the applied voltage 
Combining the Equation (8) with the Equation (1), we obtain the Equation (9) [9]:

$P=\frac{V^{2}}{R}$

Hence, we can find that the electric power $(P)$ is proportional to the square of the voltage $(V)$. For composite films with $0.01-2.0 \mathrm{wt} \%$ MWCNTs, the electric power values calculated from the Equation (8) are presented in Figure 7b. For all the composite films, the electric power increased quadratically with respect to the applied voltage in accordance with Equation (9), which demonstrates that the MWCNT/ epoxy composite films worked as a typical ohmic conductor possessing high efficiency for transforming electric energy into heat energy. The electric power $(P)$ plays a role in bridging the electric energy and heat energy with Joule's law (Equation (10)) [11]:

$Q=P t=\frac{V^{2} t}{R}$

where $Q$ is the heat generated in the conductive pathway; thus, it is reasonable to contend that the nonlinear variation in the equilibrium temperature with the applied voltage is associated with the quadratic increment in the electric power with the applied voltage.

The operational stability of the electric heating behaviour of the composite films was evaluated by conducting heating-cooling and long-term operating experiments, as shown in Figure 8. Figure 8a shows that the characteristic parameters $\left(\tau_{\mathrm{g}}\right.$ and $\left.h_{\mathrm{r}+\mathrm{c}}\right)$ and the equilibrium temperature fluctuated within a small range during the whole cyclic process, which supports the idea that the composite films have reliable, repeatable

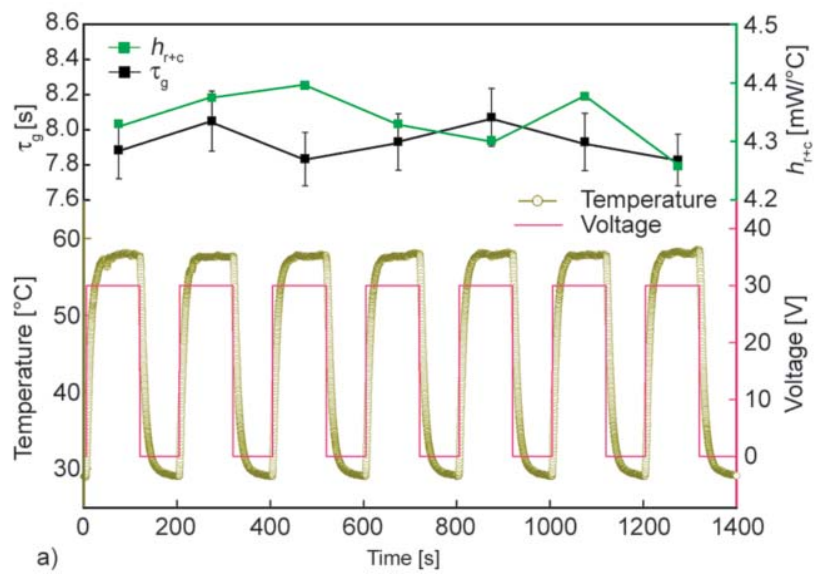

electric heating behaviour. In Figure 8b, an average temperature of $59.2 \pm 0.31^{\circ} \mathrm{C}$ was recorded for the long-term operating experiment, and the electric current of $5.17 \mathrm{~mA}$ remained constant without any obvious change over time. Therefore, the steady conductive pathway among the inter-MWCNT networks developed within the composite films did not exhibit structural changes.

\subsection{Thermal stability}

Thermal stability is one of the most important properties of conductive polymer composites as the electric heating element. The data of thermogravimetric analysis of neat epoxy and its composite specimens is shown in Table 2. It was found that the weight loss reached the maximum thermal decomposition rate at approximately $370-390{ }^{\circ} \mathrm{C}$ for the neat epoxy resin and its composite specimens. Specifically, the decomposition temperatures, $T_{5 \%}, T_{50 \%}$, and $T_{\max }$, increased slightly as the MWCNT content increased, indicating that the thermal decomposition was retarded by the presence of MWCNT in the epoxy matrix. This result was attributed to the physical barrier effect of the MWCNTs [24], which delays the extension of thermal decomposition reactions in polymer matrix. In addition, the specimens with higher MWNCT contents left more residues at $600^{\circ} \mathrm{C}$ because MWCNTs could not decompose during the heating process and were left as a residue. On the other hand, the storage modulus $\left(E^{\prime}\right)$ and loss tangent $(\tan \delta)$ versus temperature curves obtained from the dynamic mechanical analysis are illustrated in Figure 9. For the neat epoxy resin, the storage modulus decreased sharply at $\sim 100^{\circ} \mathrm{C}$ due to the glass-to-rubber transition. The peak of $\tan \delta$ at $\sim 139^{\circ} \mathrm{C}$, which originated from the

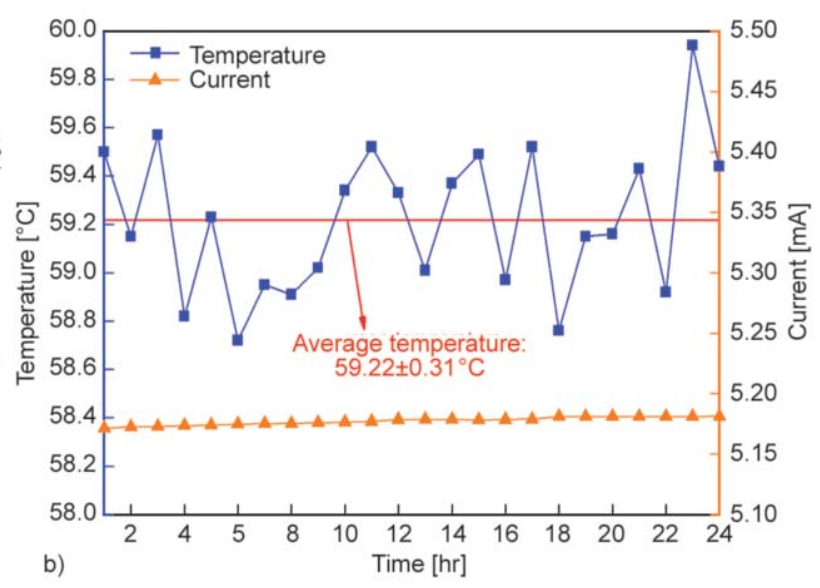

Figure 8. Operational stability tests of the composite films. (a) Temperature response of CNT/EP_0.7 under a cyclical voltage of $30 \mathrm{~V}$. (b) Time-dependent temperature curve and electric circuit value of CNT/EP_ 0.7 under an applied voltage of $30 \mathrm{~V}$. 
Table 2. Data of thermogravimetric analysis of neat epoxy and its composite specimens

\begin{tabular}{|l|c|c|c|c|}
\hline \multicolumn{1}{|c|}{ Specimens } & Epoxy & CNT/EP_0.07 & CNT/EP_0.7 & CNT/EP_1.0 \\
\hline$T_{5 \%}{ }^{1}\left[{ }^{\circ} \mathrm{C}\right]$ & 337.0 & 339.4 & 278.6 & 351.8 \\
\hline$T_{50 \%}{ }^{1}\left[{ }^{\circ} \mathrm{C}\right]$ & 382.0 & 387.0 & 387.5 & 395.0 \\
\hline$T_{\max }{ }^{2}\left[{ }^{\circ} \mathrm{C}\right]$ & 378.2 & 383.7 & 386.2 & 387.2 \\
\hline$W_{600}{ }^{3}[\%]$ & 8.23 & 8.16 & 9.66 & 11.99 \\
\hline
\end{tabular}

${ }^{1}$ Decomposition temperatures at 5 and $50 \%$ weight loss.

${ }^{2}$ Decomposition temperature at the maximum decomposition rate obtained by derivative TG (DTG).

${ }^{3}$ Residual yield at $600^{\circ} \mathrm{C}$.

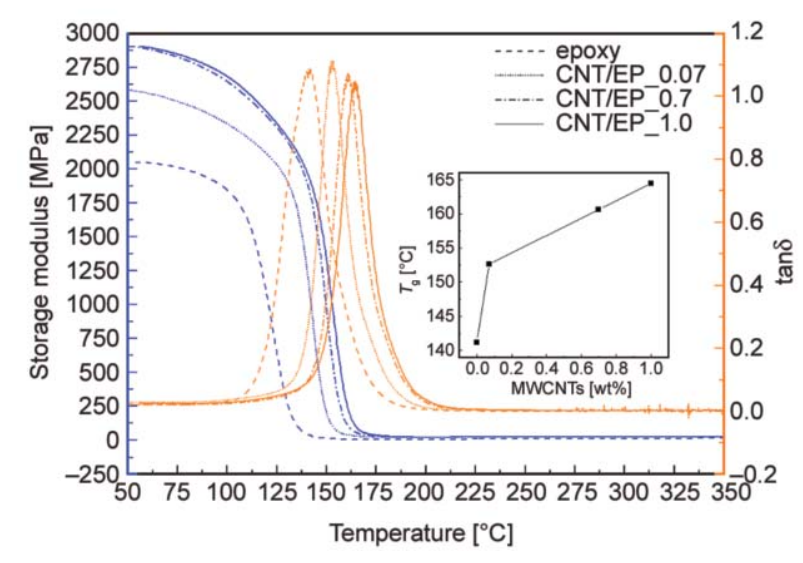

Figure 9. Dynamic storage modulus $\left(E^{\prime}\right)$ and loss tangent $(\tan \delta)$ of the neat epoxy and its composite specimens with different MWCNT contents. Inset is the glass transition temperature versus MWCNT contents curve

$\alpha$-relaxation of the epoxy molecular chains, was associated with the glass transition temperature $\left(T_{\mathrm{g}}\right)$. In the cases of the composite films, the addition of MWCNTs to epoxy resin showed an obvious influence on the storage modulus in the glassy region, and the storage modulus increased appreciably over the glassy region. It is noteworthy that the peak glass transition temperatures increased slightly as a function of MWCNT content, which was caused by the MWCNTs dispersed in the epoxy matrix restrained the mobility of epoxy chains during the glass transition. Overall, compared with the wet-fabrication method with ultrasonication [11], physical shear dispersion produced weaker interfacial interactions between the MWCNTs and epoxy resin; thus, the reinforcing effect of the MWCNTs on the polymer matrix was inferior.

\section{Conclusions}

In the present work, the MWCNT/epoxy composite films as the electric heating element were successfully prepared by a sequential process of premixing, post dispersing, film casting, and thermal curing. The effects of the shear dispersion on the microstructural feature, electrical and thermal conductivity, and electric heating behaviour were investigated. The SEM images revealed that the agglomerated MWCNTs were separated and dispersed randomly within the resin matrix and there were weak interfacial interactions between the MWCNTs and the polymer matrix. The mismatch between the gap setting of the adjacent rollers and the diameter of the MWCNTs resulted in some micron- and submicron-scale agglomeration. The electrical resistivity changed with the MWCNT content in accordance with the expected percolation behaviour, and a low percolation threshold of $\sim 0.018 \mathrm{wt} \%$ was fitted. The electric heating behaviour of the composite film was strongly dependent on the MWCNT content and applied voltage. In the thermal conductivity test, the large thermal contact resistance among the nanotube-nanotube and nanotube-matrix interfaces restricts the growth of thermal conductivity of the composite specimens. Compared with the wet-fabrication method with ultrasonication, the excellent electric heating behaviour, such as the rapid temperature response, electric heating efficiency, and operational stability, was also observed in this work. Overall, the MWCNT/epoxy composite films produced by a 3-roll mill can be applied as electric heating element with excellent electric heating behaviour and thermodynamic stability. In addition, this kind of non-solvent dispersion using a 3-roll mill is easily scalable from the laboratory to industrial manufacturing and is highly cost-efficient.

\section{Acknowledgements}

The authors are very thankful to the Chuzhou Hui-Sheng Electronic Materials Co., Ltd for their assistance of this work. The National Natural Science Foundation of China (No.11302054, 11532013), Natural Science Foundation of Heilongjiang Province of China (No.A2015012) and Fundamental Research Funds for the Central Universities (No. HEUCF170204) are acknowledged for financial support. 


\section{References}

[1] Asthana A., Maitra T., Büchel R., Tiwari M. K., Poulikakos D.: Multifunctional superhydrophobic polymer/carbon nanocomposites: Graphene, carbon nanotubes, or carbon black? ACS Applied Materials and Interfaces, 6, 8859-8867 (2014). https://doi.org/10.1021/am501649w

[2] Al-Saleh M. H., Sundararaj U.: A review of vapor grown carbon nanofiber/polymer conductive composites. Carbon, 47, 2-22 (2009).

https://doi.org/10.1016/j.carbon.2008.09.039

[3] Chatterjee S., Nafezarefi F., Tai N. H., Schlagenhauf L., Nüesch F. A., Chu B. T. T.: Size and synergy effects of nanofiller hybrids including graphene nanoplatelets and carbon nanotubes in mechanical properties of epoxy composites. Carbon, 50, 5380-5386 (2012). https://doi.org/10.1016/j.carbon.2012.07.021

[4] Song S. H., Park K. H., Kim B. H., Choi Y. W., Jun G. H., Lee D. J., Kong B. S., Paik K. W., Jeon S.: Enhanced thermal conductivity of epoxy-graphene composites by using non-oxidized graphene flakes with non-covalent functionalization. Advanced Materials, 25, 732-737 (2013).

https://doi.org/10.1002/adma.201202736

[5] Safdari M., Al-Haik M. S.: Synergistic electrical and thermal transport properties of hybrid polymeric nanocomposites based on carbon nanotubes and graphite nanoplatelets. Carbon, 64, 111-121 (2013). https://doi.org/10.1016/j.carbon.2013.07.042

[6] Tang G., Jiang Z-G., Li X., Zhang H-B., Hong S., Yu Z-Z.: Electrically conductive rubbery epoxy/diaminefunctionalized graphene nanocomposites with improved mechanical properties. Composites Part B: Engineering, 67, 564-570 (2014).

https://doi.org/10.1016/j.compositesb.2014.08.013

[7] Bauhofer W., Kovacs J. Z.: A review and analysis of electrical percolation in carbon nanotube polymer composites. Composites Science and Technology, 69, 14861498 (2009).

https://doi.org/10.1016/j.compscitech.2008.06.018

[8] An J-E., Jeong Y. G.: Structure and electric heating performance of graphene/epoxy composite films. European Polymer Journal, 49, 1322-1330 (2013). https://doi.org/10.1016/j.eurpolymj.2013.02.005

[9] Jeong Y. G., An J-E.: Effects of mixed carbon filler composition on electric heating behavior of thermally-cured epoxy-based composite films. Composites Part A: Applied Science and Manufacturing, 56, 1-7 (2014). https://doi.org/10.1016/j.compositesa.2013.09.003

[10] Tang P., Zhang R., Chen Z., Yang B., Bin Y.: Effect of $\gamma$-ray irradiation on the microstructure and self-heating property of carbon fiber/polyethylene composite films. Composites Part A: Applied Science and Manufacturing, 78, 174-180 (2015).

https://doi.org/10.1016/j.compositesa.2015.07.021

[11] Park J., Jeong Y. G.: Investigation of microstructure and electric heating behavior of hybrid polymer composite films based on thermally stable polybenzimidazole and multiwalled carbon nanotube. Polymer, 59, 102-109 (2015).

https://doi.org/10.1016/j.polymer.2015.01.003
[12] Chu K., Yun D-J., Kim D., Park H., Park S-H.: Study of electric heating effects on carbon nanotube polymer composites. Organic Electronics, 15, 2734-2741 (2014). https://doi.org/10.1016/j.orgel.2014.07.043

[13] Neitzert H. C., Vertuccio L., Sorrentino A.: Epoxy/ MWCNT composite as temperature sensor and electrical heating element. IEEE Transactions on Nanotechnology, 10, 688-693 (2011). https://doi.org/10.1109/TNANO.2010.2068307

[14] Isaji S., Bin Y., Matsuo M.: Electrical conductivity and self-temperature-control heating properties of carbon nanotubes filled polyethylene films. Polymer, 50, 1046-1053 (2009).

https://doi.org/10.1016/j.polymer.2008.12.033

[15] Choi I., Lee D., Lee D. G.: Radar absorbing composite structures dispersed with nano-conductive particles. Composite Structures, 122, 23-30 (2015).

https://doi.org/10.1016/j.compstruct.2014.11.040

[16] Thostenson E. T., Ziaee S., Chou T-W.: Processing and electrical properties of carbon nanotube/vinyl ester nanocomposites. Composites Science and Technology, 69, 801-804 (2009).

https://doi.org/10.1016/j.compscitech.2008.06.023

[17] Thostenson E. T., Chou T-W.: Processing-structure-multifunctional property relationship in carbon nanotube/ epoxy composites. Carbon, 44, 3022-3029 (2006). https://doi.org/10.1016/j.carbon.2006.05.014

[18] Gojny F. H., Wichmann M. H. G., Köpke U., Fiedler B., Schulte K.: Carbon nanotube-reinforced epoxy-composites: Enhanced stiffness and fracture toughness at low nanotube content. Composites Science and Technology, 64, 2363-2371 (2004). https://doi.org/10.1016/j.compscitech.2004.04.002

[19] Throckmorton J. A., Watters A. L., Geng X., Palmese G. R.: Room temperature ionic liquids for epoxy nanocomposite synthesis: Direct dispersion and cure. Composites Science and Technology, 86, 38-44 (2013). https://doi.org/10.1016/j.compscitech.2013.06.016

[20] Stauffer D., Aharony A.: Introduction to percolation theory. CRC Press, London (1994).

[21] Battisti A., Skordos A. A., Partridge I. K.: Percolation threshold of carbon nanotubes filled unsaturated polyesters. Composites Science and Technology, 70, 633637 (2010). https://doi.org/10.1016/j.compscitech.2009.12.017

[22] Mąka H., Spychaj T., Zenker M.: High performance epoxy composites cured with ionic liquids. Journal of Industrial and Engineering Chemistry, 31, 192-198 (2015). https://doi.org/10.1016/j.jiec.2015.06.023

[23] Park J. G., Cheng Q., Lu J., Bao J., Li S., Tian Y., Liang Z., Zhang C., Wang B.: Thermal conductivity of MWCNT/epoxy composites: The effects of length, alignment and functionalization. Carbon, 50, 20832090 (2012).

https://doi.org/10.1016/j.carbon.2011.12.046

[24] Kim J. Y., Kim S. H.: Influence of multiwall carbon nanotube on physical properties of poly(ethylene 2,6naphthalate) nanocomposites. Journal of Polymer Science Part B: Polymer Physics, 44, 1062-1071 (2006). https://doi.org/10.1002/polb.20728 